J. Dairy Sci. 98:4521-4534

http://dx.doi.org/10.3168/jds.2014-9137

(C) American Dairy Science Association ${ }^{\circledR}, 2015$.

\title{
Phenotypic antimicrobial susceptibility and occurrence of selected resistance genes in gram-positive mastitis pathogens isolated from Wisconsin dairy cows
}

\author{
P. L. Ruegg,${ }^{* 1}$ L. Oliveira, ${ }^{*}$ W. Jin, $†$ and O. Okwumabuał \\ *Department of Dairy Science, University of Wisconsin, Madison 53706 \\ †School of Veterinary Medicine, Ministry of Education Key Laboratory for Avian Preventive Medicine, Yangzhou University, Yangzhou, \\ Jiangsu 225009, China \\ $\ddagger$ School of Veterinary Medicine, Wisconsin Veterinary Diagnostic Laboratory, University of Wisconsin, Madison 53706
}

\section{ABSTRACT}

In the United States, few intramammary antimicrobials exist that are approved for treatment of bovine mastitis; thus, ensuring judicious use of these products is a priority. The objectives of this study were to determine phenotypic susceptibility and presence of selected antimicrobial resistance genes from staphylococci, streptococci, and streptococcal-like organisms recovered from cases of clinical mastitis occurring in cows on large Wisconsin farms. Staphylococcus aureus $(\mathrm{n}=35$ from 19 herds), coagulase-negative staphylococci ( $\mathrm{n}=$ 51 from 30 herds), Streptococcus spp. (n $=78$ from 36 herds), and streptococcal-like organisms ( $\mathrm{n}=31$ from 19 herds) were used in this study. All Staphylococcus spp. were susceptible to ceftiofur, cephalothin, and the combination of penicillin and novobiocin. Of all staphylococci, only a single Staphylococcus epidermidis exhibited phenotypic resistance to oxacillin. Phenotypic susceptibility to erythromycin was observed in only 8.6 and $15.7 \%$ of Staphylococcus aureus and coagulasenegative staphylococci, respectively. Approximately $20 \%$ of staphylococci and 13 to $22 \%$ of streptococci and streptococcal-like organisms exhibited phenotypic resistance to pirlimycin. All Streptococcus spp. exhibited phenotypic susceptibility to ceftiofur, cephalothin, and oxacillin. The proportion of isolates exhibiting phenotypic susceptibility to pirlimycin and sulfadimethoxine differed among Streptococcus dysgalactiae and Streptococcus uberis. All streptococcal-like organisms exhibited phenotypic susceptibility to ceftiofur, cephalothin, oxacillin, penicillin, and the combination of penicillin and novobiocin. Of all organisms tested, $36.9 \%$ did not carry any of the resistance genes (ermC, blaZ, tetK, or tet $M), 35.4 \%$ carried 1 gene, and $27.7 \%$ carried multiple

Received November 21, 2014

Accepted February 13, 2015.

${ }^{1}$ Corresponding author: plruegg@wisc.edu genes (usually blaZ in combination with a tet gene). Eighteen (51.4\%) Staph. aureus and 12 (48.0\%) Staphylococcus chromogenes carried multiple resistance genes. Six $(12.2 \%)$ Strep. dysgalactiae and no Strep. uberis carried multiple resistance genes. Results indicate that most gram-positive mastitis organisms were susceptible to most antimicrobials used for intramammary administration, but some resistance to drugs used for systemic treatment of mastitis was noted. The presence of selected resistance genes was not proportional to the occurrence of phenotypic resistance.

Key words: treatment, mastitis, dairy, resistance

\section{INTRODUCTION}

Mastitis continues to be a common disease of dairy cows and one of the most significant factors limiting dairy farm profitability. Recent studies have indicated that most cases of clinical mastitis occurring in dairy cows in developed dairy regions are caused by environmental pathogens (Bradley et al., 2007; Oliveira et al., 2013). Among gram-positive organisms, environmental streptococci and CNS are the most prevalent pathogens recovered from clinical cases of mastitis occurring on dairy farms (McDougall et al., 2007; Pinzón-Sanchez and Ruegg, 2011; Oliveira et al., 2013).

The use of antimicrobials to prevent or treat mastitis is one of the main reasons for administration of antimicrobials to dairy cows (Pol and Ruegg, 2007b; USDA, 2007; Saini et al., 2012). Use of antimicrobials in foodproducing animals is under increasing scrutiny due to concern about potential development of antimicrobial resistance. Bacterial resistance to specific antimicrobial classes may occur intrinsically due to a lack of binding sites or other pharmacological characteristics; this can cause clinical problems, but is not considered to be a major public health issue (Neu, 1992). Acquired antimicrobial resistance has the potential for transmission to humans and is of great concern to public health authorities (Neu, 1992). 
Antimicrobial resistance of mastitis pathogens is most commonly measured using phenotypic susceptibility tests that assess the ability of a range of concentrations of selected antimicrobials to inhibit bacterial growth (Walker, 2006). In veterinary medicine, the most commonly used phenotypic methods include disk diffusion, agar dilution, and broth microdilution (Walker, 2006).

Genotypic methods are used for detection of selected antimicrobial resistance genes, and hundreds of such genes have been characterized for gram-negative and gram-positive pathogens (Woodford and Sundsfjord, 2005). These methods assess the genotype of the organism, whereas phenotypic susceptibility tests asses the phenotype (or expression of the genotype) under laboratory conditions. As compared with phenotypic methods, resistance of a microorganism to a specific antimicrobial may occur via mechanisms associated with different resistance genes, whereas phenotypic methods simply detect results of gene expression (Cockerill, 1999).

Although the overall prevalence of resistance to most antimicrobials used in intramammary (IMM) compounds is reported to be low (Rajala-Schultz et al., 2004; Pol and Ruegg, 2007a; Bengtsson et al., 2009; Oliveira et al., 2012), monitoring the development of antimicrobial resistance is important to ensure the continued health of animals and humans. In addition, carriage of antimicrobial resistance genes by mastitis pathogens may be a potential source of gene transmission, indicating the importance of monitoring for selected resistance genes (Walther and Perreten, 2007; Vanderhaeghen et al., 2010). The objectives of the current study were to determine phenotypic susceptibility and presence of selected antimicrobial resistance genes from staphylococci, streptococci, and streptococcal-like organisms (SLO) recovered from cases of clinical mastitis occurring in cows on Wisconsin herds.

\section{MATERIALS AND METHODS}

\section{Enrollment of Farms and Selection of Isolates}

Recruitment of herds, enrollment of cows, and collection of data has been described previously (Oliveira et al., 2013; Oliveira and Ruegg, 2014). In brief, dairy herds were required to have a minimum of 200 lactating and dry cows (90th percentile for herd size in WI), participate in monthly DHI testing, record animal health data into computerized records, use a milking routine that included routine fore-stripping of quarters for detection of mastitis, and administer antimicrobials to treat affected cows. Data was collected between March and November 2010.
Most farms identified clinical mastitis by observation of abnormal milk or occurrence of other local or systemic symptoms. Trained milking technicians were responsible for case detection and collection of consecutive duplicate quarter milk samples from clinically affected quarters before administration of treatment. After collection of the milk sample, cows were treated according to individual farm protocol without influence by study personnel (Oliveira and Ruegg, 2014). All milk samples were frozen (median days frozen was 27 d; Oliveira and Ruegg, 2014) and mailed to University of Wisconsin-Madison Milk Quality Laboratory. All Staphylococcus spp., Streptococcus spp. and SLO identified in the original study were eligible for analysis of antimicrobial susceptibility, but only 1 isolate from each animal was used (Oliveira et al., 2013). After confirmation of the organism, Staphylococcus aureus (n $=35$ from 19 herds), CNS ( $\mathrm{n}=51$ from 30 herds), Streptococcus spp. ( $\mathrm{n}=78$ from 36 herds), and SLO ( $\mathrm{n}=31$ from 19 herds) isolated from cases of bovine clinical mastitis were used in the current study.

\section{Microbiological Procedures}

Upon arrival at the University of Wisconsin Milk Quality Laboratory, milk samples were thawed at room temperature, $100 \mu \mathrm{L}$ of milk from each duplicate sample was inoculated onto each half of a blood agar plate, and $10 \mu \mathrm{L}$ were plated onto a quarter of a MacConkey agar. Plates were incubated at $37^{\circ} \mathrm{C}$ for 24 to $48 \mathrm{~h}$. Microbiologic procedures were conducted according to National Mastitis Council guidelines (National Mastitis Council, 1999). Staphylococcus aureus was differentiated from other staphylococci by means of mannitol and tube coagulase reactions. Suspected Streptococcus spp. were identified as gram-positive, catalase-negative cocci by the Christie, Atkins, Munch-Petersen test and esculin reaction. After identification at the genus level, all isolates were frozen in $20 \%$ glycerol solution and stored at $-80^{\circ} \mathrm{C}$ until further analyses. For future analysis, frozen isolates were thawed, plated, and passed twice on blood agar plate.

Phenotypic identification of staphylococci and SLO at the species level was initially performed using API Staph and API 20 Strep System, respectively (BioMérieux Inc., Durham, NC). Final identification of staphylococci was performed using partial sequencing of the rрo $B$ gene.

\section{DNA Extraction}

Bacterial DNA was extracted using a heat lysisbased method. Four colonies from a pure 24-h culture 
of bacteria were suspended in $200 \mu \mathrm{L}$ of sterile water in a microcentrifuge tube. The samples were heated at $100^{\circ} \mathrm{C}$ for $10 \mathrm{~min}$ in a heat block, centrifuged at 13,000 $\times g$ for 2 min at room temperature to pellet cellular debris, then frozen at $-20^{\circ} \mathrm{C}$ until further analyses.

\section{Molecular Identification of Staphylococcus spp.}

For confirmation of identification of staphylococcal species, partial $r p o B$ gene sequencing was performed as described previously by Drancourt and Raoult (2002). The PCR products were purified by electroelution (Sambrook and Russell, 2001) after electrophoresis at $70 \mathrm{~V}$ for $1 \mathrm{~h}$ on a $0.7 \%$ agarose gel. The nucleotide sequences were assembled and compared with the nucleotide sequences at the GenBank database using the BLAST network service (http://blast.ncbi.nlm.nih. gov/Blast.cgi). Strains with homology of $97 \%$ or greater were characterized as the same species (Drancourt and Raoult, 2002).

\section{Determination of Phenotypic Antimicrobial Susceptibility}

Antimicrobial susceptibility was performed using a custom-designed broth microdilution panel that included the use of quality control organisms (Sensititer; Trek Diagnostic Systems Inc., Westlake, OH). The custom panel was used because previous research indicated that isolates were frequently inhibited at the least or greatest concentrations, which reduced the ability to determine the true MIC (Pol and Ruegg, 2007a; Apparao et al., 2009b; Oliveira et al., 2012). The custom panel included all 10 of the antimicrobials included in the mastitis panel of the most commonly used commercially available broth dilution testing system (Sensititer; Trek Diagnostic Systems Inc.) and added testing for enrofloxacin and florfenicol. As compared with the commercially available mastitis panel, the custom panel included an additional 3 to 7 dilutions (depending on the antimicrobial). This method was performed in accordance with guidelines established by the Clinical Laboratory Standards Institute (CLSI, 2008). Each of the 96 wells in the custom-designed extended dilution panel for MIC determination contained serial dilutions of the following 12 antimicrobial agents as described: ampicillin (0.015-2.0 $\mu \mathrm{g} / \mathrm{mL})$, ceftiofur (0.015-4.0 $\mu \mathrm{g} / \mathrm{mL})$, cephalothin $(0.03-16 \mu \mathrm{g} / \mathrm{mL})$, enrofloxacin $(0.06-1.0 \mu \mathrm{g} / \mathrm{mL})$, erythromycin $(0.015-2.0 \mu \mathrm{g} / \mathrm{mL})$, florfenicol $(0.5-16.0 \mu \mathrm{g} / \mathrm{mL})$, oxacillin $(0.12-4.0 \mu \mathrm{g} /$ $\mathrm{mL})$, penicillin $(0.015-4.0 \mu \mathrm{g} / \mathrm{mL})$, the combination of penicillin and novobiocin $(0.03-4.0 \mu \mathrm{g} / \mathrm{mL})$, pirlimycin (0.06-8.0 $\mu \mathrm{g} / \mathrm{mL})$, sulfadimethoxine $(16-1024 \mu \mathrm{g} / \mathrm{mL})$, and tetracycline $(0.06-32.0 \mu \mathrm{g} / \mathrm{mL})$. Two positive con- trol wells were also included in each panel. Isolates were subcultured twice on blood agar plates, and bacterial suspensions were prepared and standardized to a 0.5 McFarland standard per the manufacturer's instructions. Aliquots $(40 \mu \mathrm{L}$ for Staphylococcus spp. and $80 \mu \mathrm{L}$ for Streptococcus spp.) of this suspension were dispensed into each well and the plates were incubated aerobically at $36^{\circ} \mathrm{C}$ for 18 to $24 \mathrm{~h}$. Quality control was performed in accordance with the guidelines specified by the Clinical Laboratory Standards Institute (CLSI, 2008) using Staph. aureus ATCC 29213 and Enterococcus faecalis ATCC 29212 as control strains. All susceptibility results from the control strains complied with the quality control ranges.

\section{Identification of Antimicrobial Resistance Genes}

Four known antimicrobial resistance genes were tested in the current study based on antimicrobial usage in enrolled herds and expected prevalence of phenotypic resistance. Detection of ermC was performed as described previously (Sutcliffe et al., 1996; Apparao, 2008) using conventional PCR and included the use of a positive control. All isolates were tested for the presence of $e r m C$ by using primers 5'-TCAAAACATAATATAGATAAA- $3^{\prime}$ and 5'-GCTAATATTGTTTAAATCGTCAAT-3' (Sutcliffe et al., 1996).

Real-time PCR was performed to detect the presence of blaZ, tetK, and tet $M$ genes. All isolates were tested for the presence of blaZ by using primers $5^{\prime}$-ACGTCTAAAAGAACTAGGAGATAAAGTAACAA- $3^{\prime}$ and 5'-CGAAAGCAGCAGGTGTTGAA-3'. All isolates were tested for the presence of tetK by using primers 5 '-TGTTATGGGCGGATTATCTTTTACT-3' and 5'-AGCAAACTCATTCCAGAAGCAACT-3'. All isolates were tested for the presence of tet $M$ by using primers 5'-AGTGGAGCGATTACAGAATTAGGAA-3' and 5'-TCTGACGTTCTAAAAGCGTATTATCC-3'. Amplification reactions were performed in a total volume of $25 \mu \mathrm{L}$ containing $12.5 \mu \mathrm{L}$ of SYBR Green PCR Master Mix (Applied Biosystems Inc., Carlsbad, CA), $0.5 \mu \mathrm{M}$ of each primer; $6.5 \mu \mathrm{L}$ of $\mathrm{H}_{2} \mathrm{O}$, and $5 \mu \mathrm{L}$ of template. The real-time PCR detection system used was ABI 7500 Fast (Applied Biosystems Inc.). The thermal cycling profile consisted of 4 stages: stage $1=50^{\circ} \mathrm{C}$ for $2 \mathrm{~min}$; stage $2=95^{\circ} \mathrm{C}$ for $15 \mathrm{~min}$; stage $3=40$ cycles at $95^{\circ} \mathrm{C}$ for $15 \mathrm{~s}$, followed by $60^{\circ} \mathrm{C}$ for $1 \mathrm{~min}$; and stage $4=95^{\circ} \mathrm{C}$ for $15 \mathrm{~s}$. The negative control was a reaction mixture containing all reagents but no DNA template. Data analysis was performed on the ABI 7500 instrument software (Applied Biosystems Inc.) using the dissociation melt curve analysis. The positive controls for blaZ, tet $M$, and tet $K$ were designed by Integrated DNA Technologies (Integrated DNA Technologies Inc., 
Coralville, IA) by supplying them with the PCR amplicon target sequences and having them implement it into a selectable vector. Additionally, sequencing of the PCR products and comparison of the sequence results to the sequences at the GenBank database revealed $100 \%$ identity to similar genes in the database suggesting that the genes were correctly identified by the set of primers used.

\section{Definitions and Classifications}

Aerococcus spp., Enterococcus spp., and Lactococcus spp. were grouped and classified as SLO. Antimicrobial resistance was defined as the ability of a bacterial pathogen to survive exposure to a defined concentration of an antimicrobial agent (CLSI, 2008, 2013). The interpretative criteria for ceftiofur, the combination of penicillin and novobiocin, and pirlimycin were based on bovine mastitis (CLSI, 2008, 2013). The interpretative criteria for ampicillin, cephalothin (the class representative for cephapirin), erythromycin, oxacillin, penicillin, sulfadimethoxine, and tetracycline were based on human data (CLSI, 2008, 2013). The interpretative criterion for enrofloxacin and florfenicol were based on cattle respiratory disease (CLSI, 2008, 2013).

\section{Statistical Analysis}

Minimum inhibitory concentration data for each antimicrobial were summarized using the 50th $\left(\mathrm{MIC}_{50}\right)$, and 90 th $\left(\mathrm{MIC}_{90}\right)$ percentiles, as well as the proportion of isolates susceptible, intermediate (when applicable), or resistant. Statistical analyses were performed using SAS 9.3 (SAS Institute, 2011).

\section{RESULTS}

\section{Characteristics of Herds}

Characteristics of the herds $(\mathrm{n}=52)$, cases, and mastitis treatments have been previously described (Oliveira et al., 2013; Oliveira and Ruegg, 2014). In brief, herds contained between 170 to 2,728 milking cows with an average daily milk yield of $33.5 \mathrm{~kg} / \mathrm{cow}$ (range $=21.0-40.8 \mathrm{~kg} / \mathrm{cow}$ ) and bulk tank SCC of 219,000 cells $/ \mathrm{mL}$ (range $=87,000-432,000$ cells $/ \mathrm{mL}$ ). All herds used antimicrobial treatments for dry cow therapy. Almost all cases were treated using 1 of 5 IMM antimicrobials that are avaiable in the United States (Oliveira and Ruegg, 2014). Of cows that received only IMM therapy, the distribution of use of IMM products was ceftiofur $(75 \%)$, cephapirin (14\%), amoxicillin $(5 \%)$, hetacillin $(4 \%)$, and pirlimycin (3\%; Oliveira and Ruegg, 2014).

\section{Identification of Staphylococcus spp.}

Based on rpoB gene sequencing, of 86 Staphylococcus spp., the most frequently identified species were Staph. aureus (40.7\%) and Staphylococcus chromogenes (29.1\%), followed by Staphylococcus haemolyticus (8.1\%; Table 1). Compared with identification using the rpoB gene, API Staph correctly identified 58 of 86 $(67.4 \%)$ isolates. Of Staph. aureus $(\mathrm{n}=35)$ identified using the rpoB gene, only $80 \%$ were in agreement using API Staph (7 Staph. aureus were misidentified; Table 1). Of 25 Staph. chromogenes isolates identified using the rро $B$ gene, 12 gave different bacterial identification using API Staph (4 as Staphylococcus epidermidis, 3 as Staph. aureus, 3 as Staphylococcus simulans, 1 as Staphylococcus auricularis, and 1 as Staphylococcus lugdune). Of 3 Staphylococcus equorum identified using the rpoB gene, 2 were identified using API Staph as Staphylococcus sciuri and 1 as Staphylococcus xylosus. Of 7 Staph. haemolyticus isolates identified using the rpoB gene, 1 was identified using API Staph as Aerococcus viridians, 1 as Staph. aureus, 1 as Staphylococcus capitis, 1 as Staph. chromogenes, 1 as Staphylococcus saprophyticus, and 1 as Staph. simulans.

\section{Phenotypic Identification of Streptococci spp. and SLO}

Isolates were identified as Streptococcus $\mathrm{spp}$. ( $\mathrm{n}=78$; $71.6 \%)$, Lactococcus lactis $(\mathrm{n}=23 ; 21.1 \%)$, Aerococcus viridians $(\mathrm{n}=5 ; 4.6 \%)$, and Enterococcus spp. $(\mathrm{n}=3$; 2.7\%; Table 2). Of Streptococcus spp. isolated from 36 herds, the most frequently isolated species were Streptococcus dysgalactiae (62.8\%) and Streptococcus uberis (28.2\%; Table 2). Lactococcus lactis, Aerococcus viridians, and Enterococcus spp. were isolated from 14, 5, and 3 herds, respectively.

\section{Antimicrobial Susceptibility Testing}

Staphylococcus spp. All staphylococci were inhibited below the breakpoint defined for susceptibility of ceftiofur, cephalothin, and the combination of penicillin and novobiocin (Table 3). Except for 1 isolate (identified as Staph. epidermidis), all isolates were inhibited by the range of concentrations tested for oxacillin. The oxacillin-resistant Staph. epidermidis was also phenotypically resistant to ampicillin, penicillin, and tetracycline. About $80 \%$ of Staph. aureus and $>90 \%$ of CNS demonstrated in vitro susceptibility to ampicillin and penicillin (Table 3). Of Staph. aureus, only $8.6 \%$ demonstrated in vitro susceptibility to erythromycin, and $20 \%$ were not inhibited at the greatest concentration of erythromycin that was tested (Table 3). Likewise, only 
Table 1. Identification of staphylococci $(n=86)$ isolated from clinical mastitis cases $(n=86)$ occurring in cows on 37 large Wisconsin dairy herds using API Staph (BioMérieux Inc., Durham, NC) compared with rpoB gene identification

\begin{tabular}{|c|c|c|c|c|c|c|}
\hline \multirow[b]{3}{*}{ Species } & \multirow{2}{*}{\multicolumn{2}{|c|}{$\begin{array}{c}\text { rpo } B \text { gene } \\
\text { identification }\end{array}$}} & \multicolumn{4}{|c|}{ API Staph } \\
\hline & & & \multicolumn{2}{|c|}{ Correctly identified } & \multicolumn{2}{|c|}{ Misidentified } \\
\hline & No. & $\%$ & No. & $\%$ & No. & $\%$ \\
\hline Staphylococcus aureus & 35 & 40.7 & 28 & 80.0 & 7 & 20.0 \\
\hline Staphylococcus chromogenes & 25 & 29.1 & 13 & 52.0 & 12 & 48.0 \\
\hline Other staphylococci & 26 & 30.2 & 17 & 65.4 & 9 & 34.6 \\
\hline Staphylococcus auricularis & 1 & 1.2 & 1 & & 0 & \\
\hline Staphylococcus cohnii & 1 & 1.2 & 1 & & 0 & \\
\hline Staphylococcus epidermidis & 4 & 4.7 & 4 & & 0 & \\
\hline Staphylococcus equorum & 3 & 3.5 & 0 & & 3 & \\
\hline Staphylococcus haemolyticus & 7 & 8.1 & 1 & & 6 & \\
\hline Staphylococcus hyicus & 4 & 4.7 & 4 & & 0 & \\
\hline Staphylococcus sciuri & 1 & 1.2 & 1 & & 0 & \\
\hline Staphylococcus simulans & 4 & 4.7 & 4 & & 0 & \\
\hline Staphylococcus xylosus & 1 & 1.2 & 1 & & 0 & \\
\hline Total & 86 & 100.0 & 58 & 67.4 & 28 & 32.6 \\
\hline
\end{tabular}

$16 \%$ of CNS were sensitive to erythromycin and $7.8 \%$ were not inhibited at the greatest concentration tested (Table 3). However, of Staph. aureus and CNS, 71.5 and $76.5 \%$, respectively, were classified as having intermediate resistance to erythromycin. No mastitis-specific interpretive criteria were available for florfenicol, but 14.3 and $2.6 \%$ of Staph. aureus and CNS, respectively, were not inhibited by the greatest concentration of this drug. In contrast, using the interpretive criteria developed for bovine respiratory disease, almost all Staphylococcus spp. would have been considered susceptible to enrofloxacin (Table 3). About $75 \%$ of Staphylococcus

Table 2. Identification of streptococci $(\mathrm{n}=78)$ and streptococcal-like organisms $(\mathrm{n}=31)$ isolated from clinical mastitis cases $(\mathrm{n}=109)$ occurring in cows on 40 large Wisconsin dairy herds using API 20 Strep (BioMérieux Inc., Durham, NC)

\begin{tabular}{lcc}
\hline & \multicolumn{2}{c}{ API 20 Strep } \\
\cline { 2 - 3 } Species & No. & $\%$ \\
\hline Streptococcus spp. & 78 & 71.6 \\
Streptococcus dysgalactiae & 49 & $62.8^{1}$ \\
Streptococcus uberis & 22 & 28.2 \\
Other streptococci & & \\
Streptococcus mitis & 2 & 2.6 \\
Streptococcus bovis & 3 & 3.8 \\
Streptococcus intermedius & 1 & 1.3 \\
Streptococcus suis & 1 & 1.3 \\
Streptococcal-like organisms & 31 & 28.4 \\
Aerococcus viridians & 5 & $16.1^{2}$ \\
Enterococcus durans & 1 & 3.2 \\
Enterococcus faecium & 2 & 6.5 \\
Lactococcus lactis & 23 & 74.2 \\
Total & 109 & 100.0 \\
\hline Dis
\end{tabular}

${ }^{1}$ Denominator is total streptococci.

${ }^{2}$ Denominator is total streptococcal-like organisms. spp. were sensitive to pirlimycin, but $20 \%$ of Staph. aureus and $10 \%$ of CNS were not inhibited at the greatest concentration of pirlimycin that was tested. A greater proportion of Staph. aureus (97\%) were sensitive to sulfadimethoxine as compared with CNS $(79 \% ; P=0.01)$, and $14 \%$ of CNS were not inhibited at the greatest concentration of sulfadimethoxine (Table 3). Around 70 to $80 \%$ of Staph. aureus and CNS demonstrated in vitro susceptibility to tetracycline

For Staph. aureus, the $\mathrm{MIC}_{50}$ and $\mathrm{MIC}_{90}$ of ceftiofur, cephalothin, enrofloxacin, oxacillin, the combination of penicillin and novobiocin, and sulfadimethoxine were either identical or within 1 or 2 dilutions. The difference in the $\mathrm{MIC}_{50}$ and $\mathrm{MIC}_{90}$ was 5 to 6 dilutions for ampicillin, penicillin, and tetracycline. The $\mathrm{MIC}_{90}$ could not be determined for erythromycin, florfenicol, or pirlimycin because $>10 \%$ of Staph. aureus were not inhibited at the greatest concentration tested (Table 3 ). For CNS, the $\mathrm{MIC}_{50}$ and $\mathrm{MIC}_{90}$ for ampicillin, ceftiofur, cephalothin, enrofloxacin, erythromycin, florfenicol, oxacillin, and the combination of penicillin and novobiocin were either identical or within 1or 2 dilutions. The differences between the $\mathrm{MIC}_{50}$ and $\mathrm{MIC}_{90}$ were 3, 4 , and 7 dilutions for penicillin, pirlimycin, and tetracycline, respectively. The $\mathrm{MIC}_{90}$ could not be determined for sulfadimethoxine because $13.7 \%$ of CNS were not inhibited at the greatest concentration tested (Table 3).

Streptococcus spp. and SLO. Almost all (90$100 \%$ Streptococcus spp. and SLO were inhibited by the range of concentrations tested for ampicillin, ceftiofur, cephalothin, erythromycin, oxacillin, penicillin, and the combination of penicillin and novobiocin (Table 3). Few Streptococcus spp. and SLO were sensitive to enrofloxacin, but using the bovine respiratory 


\begin{tabular}{|c|c|c|c|c|c|c|c|c|c|c|c|c|c|c|c|c|c|c|c|c|c|c|}
\hline \multirow{2}{*}{ Antimicrobial } & \multirow{2}{*}{$\begin{array}{c}\mathrm{BP}^{2} \\
(\mathrm{ug} / \mathrm{mL})\end{array}$} & \multirow[b]{2}{*}{ Etiology } & \multirow[b]{2}{*}{ No. } & \multirow{2}{*}{$\begin{array}{l}\mathrm{S}^{3} \\
(\%)\end{array}$} & \multicolumn{18}{|c|}{ Percentage of isolates at each indicated $\mathrm{MIC}^{4}(\mu \mathrm{g} / \mathrm{mL})$} \\
\hline & & & & & 0.015 & 0.03 & 0.06 & 0.12 & 0.25 & 0.5 & 1 & 2 & 4 & 8 & 16 & 32 & 64 & 128 & 256 & 512 & 1024 & $\mathrm{NI}^{5}$ \\
\hline \multirow[t]{4}{*}{ Ampicillin } & $\leq 0.25$ & $\begin{array}{l}\text { Staph. } \\
\text { aureus }\end{array}$ & 35 & 77 & $\underline{57.1}$ & 5.7 & 8.6 & 5.7 & 0.0 & 14.3 & 8.6 & 0.0 & $-^{6}$ & - & - & - & - & - & - & - & - & 0.0 \\
\hline & $\leq 0.25$ & $\mathrm{CNS}^{7}$ & 51 & 92 & 27.5 & $\underline{35.3}$ & 7.8 & 21.6 & 0.0 & 5.9 & 0.0 & 2.0 & - & - & - & - & - & - & - & - & - & 0.0 \\
\hline & $\leq 0.25$ & Strep. spp. & 78 & 97 & $\underline{55.1}$ & 5.1 & 20.5 & 15.4 & 1.3 & 2.6 & 0.0 & 0.0 & - & - & - & - & - & - & - & - & - & 0.0 \\
\hline & $\leq 8.00^{8}$ & SLO & 31 & 97 & 3.2 & 6.5 & 19.4 & $\underline{58.1}$ & 9.7 & 0.0 & 0.0 & 3.2 & - & - & - & - & - & - & - & - & - & 0.0 \\
\hline \multirow[t]{4}{*}{ Ceftiofur } & $\leq 2.00$ & $\begin{array}{l}\text { Staph. } \\
\text { aureus }\end{array}$ & 35 & 100 & 2.9 & 0.0 & 0.0 & $\overline{17.1}$ & $\underline{31.4}$ & 37.1 & 11.4 & 0.0 & 0.0 & - & - & - & - & - & - & - & - & 0.0 \\
\hline & & CNS & 51 & 100 & 0.0 & 0.0 & 0.0 & 9.8 & 31.4 & $\underline{39.2}$ & 17.7 & 2.0 & 0.0 & - & - & - & - & - & - & - & - & 0.0 \\
\hline & & Strep. spp. & 78 & 100 & $\underline{52.6}$ & 27.0 & 12.8 & 5.1 & 2.6 & $\overline{0.0}$ & 0.0 & 0.0 & 0.0 & - & - & - & - & - & - & - & - & 0.0 \\
\hline & & SLO & 31 & 100 & 0.0 & 32.3 & $\underline{45.2}$ & 6.5 & 12.9 & 0.0 & 3.2 & 0.0 & 0.0 & - & - & - & - & - & - & - & - & 0.0 \\
\hline \multirow[t]{4}{*}{ Cephalothin } & $\leq 8.00$ & $\begin{array}{l}\text { Staph. } \\
\text { aureus }\end{array}$ & 35 & 100 & - & 14.3 & 20.0 & $\underline{37.1}$ & 14.3 & 5.7 & 5.7 & 0.0 & 0.0 & 2.9 & 0.0 & - & - & - & - & - & - & 0.0 \\
\hline & & CNS & 51 & 100 & - & 2.0 & 25.5 & 47.1 & 15.7 & 7.8 & 2.0 & 0.0 & 0.0 & 0.0 & 0.0 & - & - & - & - & - & - & 0.0 \\
\hline & & Strep. spp. & 78 & 100 & - & 0.0 & 29.5 & $\overline{20.5}$ & 15.4 & 14.1 & 5.1 & 11.5 & 3.9 & 0.0 & 0.0 & - & - & - & - & - & - & 0.0 \\
\hline & & SLO & 31 & 100 & - & 0.0 & 3.2 & 0.0 & 3.2 & 6.5 & 0.0 & $\underline{58.1}$ & 22.6 & 6.5 & 0.0 & - & - & - & - & - & - & 0.0 \\
\hline \multirow[t]{4}{*}{ Enrofloxacin } & $<0.25^{9}$ & $\begin{array}{l}\text { Staph. } \\
\text { aureus }\end{array}$ & 35 & 97 & - & - & 5.7 & 42.9 & $\underline{48.57}$ & 2.9 & 0.0 & - & - & - & - & - & - & - & - & - & - & 0.0 \\
\hline & & CNS & 51 & 98 & - & - & 9.8 & 62.8 & 25.5 & 2.0 & 0.0 & - & - & - & - & - & - & - & - & - & - & 0.0 \\
\hline & & Strep. spp. & 78 & 9 & - & - & 2.6 & $\overline{1.3}$ & 5.1 & $\underline{50.0}$ & 33.0 & - & - & - & - & - & - & - & - & - & - & 7.7 \\
\hline & & SLO & 31 & 10 & - & - & 3.2 & 3.2 & 3.2 & 22.6 & $\underline{64.5}$ & - & - & - & - & - & - & - & - & - & - & 3.2 \\
\hline \multirow[t]{4}{*}{ Erythromycin } & $\leq 0.50$ & $\begin{array}{l}\text { Staph. } \\
\text { aureus }\end{array}$ & 35 & 9 & 2.9 & 0.0 & 0.0 & 0.0 & 0.0 & 5.7 & $\underline{\underline{68.6}}$ & 2.9 & - & - & - & - & - & - & - & - & - & 20.0 \\
\hline & $\leq 0.50$ & CNS & 51 & 16 & 0.0 & 0.0 & 0.0 & 0.0 & 0.0 & 15.7 & $\underline{60.8}$ & 15.7 & - & - & - & - & - & - & - & - & - & 7.8 \\
\hline & $\leq 0.25$ & Strep. spp. & 78 & 95 & 2.6 & 0.0 & 16.7 & $\underline{64.1}$ & 11.5 & 0.0 & 0.0 & 3.9 & - & - & - & - & - & - & - & - & - & 1.3 \\
\hline & $\leq 0.50^{8}$ & SLO & 31 & 90 & 0.0 & 0.0 & 3.2 & $\overline{54.8}$ & 32.2 & 9.7 & 0.0 & 0.0 & - & - & - & - & - & - & - & - & - & 0.0 \\
\hline \multirow[t]{4}{*}{ Florfenicol } & $<2.0^{9}$ & $\begin{array}{l}\text { Staph. } \\
\text { aureus }\end{array}$ & 35 & 37 & - & - & - & - & - & 0.0 & 2.9 & 34.3 & $\underline{48.6}$ & 0.0 & 0.0 & - & - & - & - & - & - & 14.3 \\
\hline & & CNS & 51 & 65 & - & - & - & - & - & 0.0 & 5.9 & $\underline{58.8}$ & 23.5 & 2.0 & 0.0 & - & - & - & - & - & - & 2.6 \\
\hline & & Strep. spp. & 78 & 97 & - & - & - & - & - & 5.1 & 44.9 & $\overline{47.4}$ & 2.6 & 0.0 & 0.0 & - & - & - & - & - & - & 0.0 \\
\hline & & SLO & 31 & 61 & - & - & - & - & - & 0.0 & 3.2 & $\underline{58.1}$ & 35.5 & 0.0 & 3.2 & - & - & - & - & - & - & 0.0 \\
\hline \multirow[t]{4}{*}{ Oxacillin } & $\leq 2.00$ & $\begin{array}{l}\text { Staph. } \\
\text { aureus }\end{array}$ & 35 & 100 & - & - & - & $\underline{100.0}$ & 0.0 & 0.0 & 0.0 & $\overline{0.0}$ & 0.0 & - & - & - & - & - & - & - & - & 0.0 \\
\hline & $\leq 0.25$ & CNS & 51 & 98 & - & - & - & $\underline{88.2}$ & 7.8 & 2.0 & 0.0 & 0.0 & 0.0 & - & - & - & - & - & - & - & - & 0.0 \\
\hline & $\leq 2.00$ & Strep. spp & 78 & 100 & - & - & - & $\overline{77.0}$ & 3.9 & 15.4 & 3.9 & 0.0 & 0.0 & - & - & - & - & - & - & - & - & 0.0 \\
\hline & & SLO & 31 & 100 & - & - & - & 12.9 & 6.5 & 45.1 & 16.1 & 19.4 & 0.0 & - & - & - & - & - & - & - & - & 0.0 \\
\hline \multirow[t]{4}{*}{ Penicillin } & $\leq 0.12$ & $\begin{array}{l}\text { Staph. } \\
\text { aureus }\end{array}$ & 35 & 80 & $\underline{71.4}$ & 0.0 & 5.7 & 2.9 & 5.7 & 8.6 & 0.0 & 0.0 & 5.7 & - & - & - & - & - & - & - & - & 0.0 \\
\hline & $<0.12$ & CNS & 51 & 90 & 62.8 & 0.0 & 17.7 & 9.8 & 2.0 & 0.0 & 0.0 & 3.9 & 0.0 & - & - & - & - & - & - & - & - & 3.9 \\
\hline & $\leq 0.12$ & Strep. spp. & 78 & 91 & $\overline{48.7}$ & 6.4 & 26.9 & 9.0 & 9.0 & 0.0 & 0.0 & 0.0 & 0.0 & & & & & & & & & 0.0 \\
\hline & $\leq 8.00^{8}$ & SLO & 31 & 100 & 3.2 & $\overline{3.2}$ & 16.1 & 19.4 & $\underline{54.8}$ & 0.0 & 0.0 & 3.2 & 0.0 & - & - & - & - & - & - & - & - & 0.0 \\
\hline \multirow[t]{4}{*}{$\begin{array}{l}\text { Penicillin- } \\
\text { novobiocin }\end{array}$} & $\leq 1 / 2$ & $\begin{array}{l}\text { Staph. } \\
\text { aureus }\end{array}$ & 35 & 100 & - & $\underline{94.3}$ & 2.9 & 0.0 & 2.9 & 0.0 & 0.0 & 0.0 & 0.0 & - & - & - & - & - & - & - & - & 0.0 \\
\hline & & CNS & 51 & 100 & - & 100.0 & 0.0 & 0.0 & 0.0 & 0.0 & 0.0 & 0.0 & 0.0 & - & - & - & - & - & - & - & - & 0.0 \\
\hline & & Strep. spp. & 78 & 99 & - & $\underline{64.1}$ & 23.1 & 9.0 & 2.6 & 1.3 & 0.0 & 0.0 & 0.0 & - & - & - & - & - & - & - & - & 0.0 \\
\hline & & SLO & 31 & 100 & - & 9.7 & 35.5 & $\underline{48.4}$ & 6.5 & 0.0 & 0.0 & 0.0 & 0.0 & - & - & - & - & - & - & - & - & 0.0 \\
\hline
\end{tabular}


Table 3 (Continued). Distribution of MIC for Staphylococcus aureus $(\mathrm{n}=35)$, CNS $(\mathrm{n}=51)$, Streptococcus $\mathrm{spp} .(\mathrm{n}=78)$ and streptococcal-like organisms $\left(\mathrm{SLO}{ }^{1} ; \mathrm{n}=31\right)$ isolated from clinical mastitis cases $(\mathrm{n}=195)$ occurring in cows on 37 large Wisconsin dairy herds

\begin{tabular}{|c|c|c|c|c|c|c|c|c|c|c|c|c|c|c|c|c|c|c|c|c|c|c|}
\hline \multirow[b]{2}{*}{ Antimicrobial } & \multirow{2}{*}{$\begin{array}{c}\mathrm{BP}^{2} \\
(\mathrm{ug} / \mathrm{mL})\end{array}$} & \multirow[b]{2}{*}{ Etiology } & \multirow[b]{2}{*}{ No. } & \multirow{2}{*}{$\begin{array}{l}\mathrm{S}^{3} \\
(\%)\end{array}$} & \multicolumn{18}{|c|}{ Percentage of isolates at each indicated $\mathrm{MIC}^{4}(\mu \mathrm{g} / \mathrm{mL})$} \\
\hline & & & & & 0.015 & 0.03 & 0.06 & 0.12 & 0.25 & 0.5 & 1 & 2 & 4 & 8 & 16 & 32 & 64 & 128 & 256 & 512 & 1024 & $\mathrm{NI}^{5}$ \\
\hline \multirow[t]{4}{*}{ Pirlimycin } & \multirow[t]{4}{*}{$\leq 2.00$} & $\begin{array}{l}\text { Staph. } \\
\text { aureus }\end{array}$ & 35 & 77 & & & 0.0 & 0.0 & 8.6 & 57.1 & 5.7 & 5.7 & 2.9 & 0.0 & & & & & & & & 20.0 \\
\hline & & CNS & 51 & 75 & - & - & 2.0 & 2.0 & 5.9 & $\underline{43.1}$ & 15.7 & 5.9 & 3.9 & 11.8 & - & - & - & - & - & - & - & 9.8 \\
\hline & & Strep. spp. & 78 & 81 & - & - & 3.9 & 34.6 & 12.8 & $\overline{16.7}$ & 9.0 & 3.9 & 0.0 & 6.4 & - & - & - & - & - & - & - & 12.8 \\
\hline & & SLO & 31 & 71 & - & - & 3.2 & 6.5 & 6.5 & 32.3 & 19.4 & 3.2 & 6.5 & 0.0 & - & - & - & - & - & - & - & 22.6 \\
\hline \multirow[t]{4}{*}{ Sulfadimethoxine } & \multirow[t]{4}{*}{$\leq 256.0$} & $\begin{array}{l}\text { Staph. } \\
\text { aureus }\end{array}$ & 35 & 97 & - & - & - & - & - & - & - & - & - & - & $\underline{77.1}$ & 17.1 & 2.9 & 0.0 & 0.0 & 2.9 & 0.0 & 0.0 \\
\hline & & CNS & 51 & 79 & - & - & - & - & - & - & - & - & - & - & 45.1 & $\underline{9.8}$ & 5.9 & 17.7 & 0.0 & 3.9 & 3.9 & 13.7 \\
\hline & & Strep. spp. & 78 & 54 & - & - & - & - & - & - & - & - & - & - & 5.1 & 11.5 & 7.7 & 29.5 & 10.3 & 0.0 & 2.6 & 33.3 \\
\hline & & SLO & 31 & 42 & - & - & - & - & - & - & - & - & - & - & 32.3 & 0.0 & 0.0 & 9.7 & 0.0 & 0.0 & 0.0 & $\underline{58.1}$ \\
\hline \multirow[t]{4}{*}{ Tetracycline } & $\leq 4.00$ & $\begin{array}{l}\text { Staph. } \\
\text { aureus }\end{array}$ & 35 & 83 & - & - & 5.7 & $\underline{65.7}$ & 2.9 & 0.0 & 2.9 & 0.0 & 5.7 & 8.6 & 2.9 & 5.7 & - & - & - & - & - & 0.0 \\
\hline & $\leq 4.00$ & CNS & 51 & 74 & - & - & 9.8 & 39.2 & $\underline{5.9}$ & 3.9 & 5.9 & 3.9 & 3.9 & 0.0 & 7.8 & 17.7 & - & - & - & - & - & 1.0 \\
\hline & \multirow[t]{2}{*}{$\leq 2.00$} & Strep. spp. & 78 & 51 & & & 3.9 & 2.6 & 5.1 & 2.6 & $\underline{34.6}$ & 2.6 & 2.6 & 1.3 & 9.0 & 33.3 & & & & & & 2.6 \\
\hline & & SLO & 31 & 71 & - & - & 0.0 & 12.9 & 25.8 & 19.4 & 6.5 & 6.5 & 0.0 & 0.0 & 9.7 & 12.9 & - & - & - & - & - & 6.5 \\
\hline
\end{tabular}

${ }^{1}$ Lactococcus lactis, Aerococcus viridians, and Enterococcus spp.

${ }^{2} \mathrm{BP}=$ breakpoint, MIC at which an isolate is considered susceptible according to CLSI (2008) guideline .

${ }^{3} \mathrm{~S}=$ percent of susceptible isolates according to CLSI (2008).

${ }^{4}$ Dash indicates values not tested for the indicated antimicrobial; $\mathrm{MIC}_{50}$ value is underlined; $\mathrm{MIC}_{90}$ value is in bold; an underlined and bold value is both the $\mathrm{MIC}_{50}$ and $\mathrm{MIC}_{90}$.

${ }^{5}$ Isolates that were not inhibited at the highest concentration of the antimicrobial tested.

${ }^{6}$ Staphylococcus chromogenes.

${ }^{7}$ Staphylococcus chromogenes, Staphylococcus auricularis, Staphylococcus cohnii, Staphylococcus epidermidis, Staphylococcus equorum, Staphylococcus haemolyticus, Staphylococcus hyicus, Staphylococcus sciuri, Staphylococcus simulans, and Staphylococcus xylosus.

${ }^{8}$ Breakpoint for enterococci.

${ }^{9}$ No breakpoints for mastitis organisms exist; breakpoint is for bovine respiratory disease. 
Table 4. Distribution of resistance genes and combinations of resistance genes in Staphylococcus aureus $(\mathrm{n}=35), \mathrm{CNS}^{1}(\mathrm{n}=51)$, Streptococcus spp. $(\mathrm{n}=78)$, and streptococcal-like organisms ${ }^{2}(\mathrm{SLO}, \mathrm{n}=31)$ isolated from clinical mastitis samples occurring in cows on 37 large Wisconsin dairy herds

\begin{tabular}{|c|c|c|c|c|c|c|c|c|c|c|c|c|}
\hline Etiology & No. & $\begin{array}{l}\text { No. }(\%) \\
\text { of genes } \\
\text { detected }\end{array}$ & \multicolumn{4}{|c|}{ Single gene } & \multicolumn{6}{|c|}{ Contains multiple genes } \\
\hline Staph. aureus & 35 & $8(23)$ & 0 & 5 & 3 & 1 & 2 & 5 & 3 & 0 & 1 & 7 \\
\hline CNS & 51 & $16(31)$ & 1 & 4 & 7 & 1 & 0 & 7 & 6 & 1 & 2 & 6 \\
\hline Strep. spp. & 78 & $37(47)$ & 0 & 9 & 6 & 17 & 0 & 3 & 2 & 2 & 0 & 2 \\
\hline
\end{tabular}

${ }^{1}$ Staphylococcus chromogenes, Staphylococcus auricularis, Staphylococcus cohnii, Staphylococcus epidermidis, Staphylococcus equorum, Staphylococcus haemolyticus, Staphylococcus hyicus, Staphylococcus sciuri, Staphylococcus simulans, and Staphylococcus xylosus.

${ }^{2}$ Lactococcus lactis, Aerococcus viridians, and Enterococcus spp.

disease breakpoints most would have been characterized as having intermediate sensitivity. Whereas most Streptococcus spp. were sensitive to florfenicol, only $61 \%$ of SLO were sensitive this compound. Overall, $81 \%$ of Streptococcus spp. were sensitive to pirlimycin as compared with $71 \%$ of SLO (Table 3). However, the distribution of sensitivity to pirlimycin among species was $83.7 \%$ (Strep. dysgalactiae), $68.2 \%$ Strep. uberis, $100 \%$ (other Streptococcus spp.), and 71\% (SLO). A large proportion of Streptococcus spp. and SLO were resistant to sulfadimethoxine (Table 3). The greatest concentration of sulfadimethoxine that was tested failed to inhibit 33.3 and $58.1 \%$ of Streptococcus spp. and SLO, respectively (Table 3). A greater proportion of Strep. dysgalactiae ( $81.6 \%$ ) were sensitive to sulfadimethoxine as compared with Strep. uberis $(31.8 \%$; $P<$ 0.001). Approximately $50 \%$ of the Streptococcus spp. and $71 \%$ of SLO demonstrated in vitro susceptibility to tetracycline (Table 3). Differences in the proportion of isolates sensitive to tetracycline were not observed for Strep. dysgalactiae or Strep. uberis.

For Streptococcus spp. and SLO, the $\mathrm{MIC}_{50}$ and $\mathrm{MIC}_{90}$ for ceftiofur, enrofloxacin, erythromycin, florfenicol, oxacillin, and the combination of penicillin and novobiocin were either the same or within 1 to 2 dilutions. For both cephalothin and penicillin, the differences in the $\mathrm{MIC}_{50}$ and $\mathrm{MIC}_{90}$ were 3 to 4 dilutions for Streptococcus spp. and 0 to 1 dilution for SLO. The $\mathrm{MIC}_{90}$ of pirlimycin could not be determined for either Streptococcus spp. or SLO because $>10 \%$ of the isolates were not inhibited at the greatest concentration tested (Table 3). Neither the $\mathrm{MIC}_{50}$ nor $\mathrm{MIC}_{90}$ of sulfadimethoxine for SLO could be determined because $58.1 \%$ of organisms were not inhibited by the greatest concentration tested. The differences between the $\mathrm{MIC}_{50}$ and $\mathrm{MIC}_{90}$ of tetracycline were 5 and 6 dilutions for Streptococcus spp. and SLO, respectively.

\section{Detection of Antimicrobial Resistance Genes}

Staphylococcus spp. Of 86 Staphylococcus spp., blaZ was detected (either alone or in combination with other genes) most frequently ( $\mathrm{n}=46$ isolates), followed by $\operatorname{tetK}(\mathrm{n}=41)$, tetM $(\mathrm{n}=26)$, and $\operatorname{erm} \mathrm{C}(\mathrm{n}=6$; Table 4). Of Staphylococcus spp., $62(72.1 \%)$ carried at least 1 of the selected resistance genes. Eight (22.8\%) Staph. aureus and 16 CNS (31.4\%) carried no resistance genes (Table 4). Eighteen (51.4\%) Staph. aureus carried multiple resistance genes. Of these isolates, 5 carried blaZ $Z$ and tetK and 7 carried blaZ, tetK, and tet $M$ (Table 4). Twelve (48.0\%) Staph. chromogenes carried multiple resistance genes. Of these isolates, 4 carried blaZ, tetK, and tetM. A similar proportion $(42.3 \%)$ of the other CNS species carried multiple resistance genes. Of these isolates, 4 carried blaZ and tet $M$ and 3 carried blaZ, tetK, and tet $M$ (Table 4).

Streptococcus spp. and SLO. Of 78 Streptococcus spp., $41(52.6 \%)$ carried at least 1 of the selected resistance genes. We detected tetM (either alone or in combination with other genes) most frequently ( $\mathrm{n}=$ 23 isolates), followed by bla $Z$ and tetK, which were detected in 16 and 13 isolates, respectively (Table 4). No Streptococcus spp. carried the ermC gene. None of the selected resistance genes were found in 21 of $49(42.8 \%)$ Strep. dysgalactiae or 16 of $22(72.7 \%)$ Strep. uberis. At least one of the selected resistance genes was identified in all of the 7 other species of streptococci. Six (12.2\%) Strep. dysgalactiae carried multiple resistance genes. Of these isolates, 2 carried blaZ and tetM, 2 carried tetK and tet $M, 1$ carried blaZ and tetK, and 1 carried blaZ, tetK and tetM. No Strep. uberis carried multiple genes.

Of 31 SLO, $20(64.5 \%)$ carried at least 1 of the selected resistance genes (Table 4). We detected tetK (either alone or in combination with other genes) most frequently $(\mathrm{n}=12)$, followed by tet $M(\mathrm{n}=9)$ and blaZ 
Table 5. Comparison of phenotypic and genotypic antimicrobial resistance for selected genes in Staphylococcus aureus $(\mathrm{n}=35)$, CNS ${ }^{1}(\mathrm{n}=$ $51)$, Streptococcus spp. $(\mathrm{n}=78)$, and streptococcal-like organisms ${ }^{2}(\mathrm{SLO} ; \mathrm{n}=31$ ) isolated from clinical mastitis samples occurring in cows on 37 large Wisconsin dairy herds

\begin{tabular}{|c|c|c|c|c|c|c|c|c|c|c|}
\hline Organism & $\begin{array}{l}\text { Susceptibility } \\
\text { test category }\end{array}$ & \multicolumn{2}{|c|}{ Erythromycin } & \multicolumn{2}{|c|}{ Pirlimycin } & \multicolumn{2}{|c|}{ Penicillin } & \multicolumn{3}{|c|}{ Tetracycline } \\
\hline \multirow[t]{3}{*}{ Staph. aureus $(\mathrm{n}=35)$} & Susceptible & 3 & $0(0)$ & 27 & $1(4)$ & 28 & $14(50)$ & 29 & $13(45)$ & $9(31)$ \\
\hline & Intermediate & 25 & $1(4)$ & $-^{3}$ & & - & & 3 & $3(100)$ & $1(33)$ \\
\hline & Resistant $^{4}$ & 7 & $2(29)$ & 8 & $2(25)$ & 7 & $7(100)$ & 3 & $2(67)$ & $1(33)$ \\
\hline \multirow{3}{*}{ Strep. spp. $(\mathrm{n}=78)$} & Susceptible & 74 & $0(0)$ & 63 & $0(0)$ & 71 & $15(21)$ & 40 & $7(18)$ & $5(13)$ \\
\hline & Intermediate & 0 & & - & & - & & 2 & $1(50)$ & $0(0)$ \\
\hline & Resistant & 4 & $0(0)$ & 15 & $0(0)$ & 7 & $2(29)$ & 36 & $4(14)$ & $18(50)$ \\
\hline \multirow[t]{3}{*}{$\mathrm{SLO}(\mathrm{n}=31)$} & Susceptible & 28 & $0(0)$ & 22 & $0(0)$ & 31 & $4(13)$ & 22 & $9(41)$ & $5(23)$ \\
\hline & Intermediate & 3 & $0(0)$ & - & & - & & 0 & & \\
\hline & Resistant & 0 & & 9 & $0(0)$ & 0 & & 9 & $3(33)$ & $4(44)$ \\
\hline
\end{tabular}

${ }^{1}$ Staphylococcus chromogenes, Staphylococcus auricularis, Staphylococcus cohnii, Staphylococcus epidermidis, Staphylococcus equorum, Staphylococcus haemolyticus, Staphylococcus hyicus, Staphylococcus sciuri, Staphylococcus simulans, and Staphylococcus xylosus.

${ }^{2}$ Lactococcus lactis, Aerococcus viridians, and Enterococcus spp.

${ }^{3}$ Organisms are not classified in this category.

${ }^{4}$ Includes organisms that were not inhibited at the greatest concentration tested.

$(\mathrm{n}=4)$. No SLO carried the ermC gene. Seven SLO carried tetK or tetM. Of 5 SLO that carried multiple resistance genes, 3 carried blaZ and tet $K$ and 2 carried tetK and tet $M$ (Table 4).

Comparison of Phenotypic Resistance and Occurrence of Resistance Genes. The proportion of isolates with phenotypic resistance did not correspond with the proportion of isolates identified with the selected resistance genes (Table 5). No Staph. aureus that demonstrated phenotypic susceptibility to erythromycin carried the erm $C$ gene; however, erm $C$ was identified in 1 of 7 phenotypically susceptible CNS (a Staph. chromogenes) (Table 5). We also detected ermC in 2 of 7 Staph. aureus and 1 of 4 CNS that were phenotypically resistant to erythromycin; all of these isolates were not inhibited by the greatest concentration of erythromycin that was tested. Likewise, ermC was detected in $25 \%$ of Staph. aureus and $8 \%$ of CNS that were phenotypically resistant to pirlimycin (Table 5). Two CNS and 1 Staph. aureus that demonstrated in vitro susceptibility to pirlimycin carried the ermC gene (Table 5). In contrast, all Staphylococcus spp. that demonstrated phenotypic resistance to penicillin $(\mathrm{n}=$ 12) carried blaZ. About 40 to $50 \%$ of staphylococci that were phenotypically susceptible to penicillin carried the blaZ gene (Table 5). Only 3 Staph. aureus demonstrated phenotypic resistance to tetracycline; tet $K$ and tet $M$ were identified in 2 and 1 of the resistant isolates, respectively (Table 5). Of 14 CNS that were resistant to tetracycline, tetK and tet $M$ were identified in $8(57 \%)$ and $3(21 \%)$, respectively. These same genes were found in about 30 to $45 \%$ of staphylococci that exhibited phenotypical susceptibility to tetracycline (Table 5).

The gene $e r m C$ was not identified in any streptococci or SLO. Of 71 streptococci that were phenotypically susceptible to penicillin, bla $Z$ was detected in 15 (21\%) (Table 5). Of 7 streptococci that were phenotypically resistant to penicillin, blaZ was found in $2(29 \%$; Table 5). We identified blaZ in 7 of $44(16 \%)$ and 1 of $21(4.8 \%)$ Strep. dysgalactiae and Strep. uberis that exhibited phenotypic susceptibility to penicillin, respectively. Based on the use of the enterococci breakpoints, all SLO were considered to be susceptible to penicillin and bla $Z$ was recovered from $13 \%$ of this group of organisms. Overall, the selected tetracycline resistance genes were identified in 13 to $18 \%$ of phenotypically susceptible streptococci, in contrast to 14 to $50 \%$ of phenotypically resistant streptococci (Table 5). Of Strep. dysgalactiae $(\mathrm{n}=22)$ that were phenotypically resistant to tetracycline, 3 and 12 isolates carried tetK and tet $M$, respectively. Of Strep. uberis $(\mathrm{n}=10)$ that demonstrated phenotypic resistance to tetracycline, tet $M$ was identified in 3 organisms and no tetK was identified. The proportion of SLO that carried either tet $K$ or tet $M$ was about the same (23-44\%) for both phenotypically susceptible and resistant organisms (Table 5).

\section{DISCUSSION}

The dairy farms enrolled in our study were reasonably representative of larger Wisconsin dairy herds that contribute the majority of milk used in processing. The 
mastitis pathogens (Oliveira et al., 2013) and treatments (Oliveira and Ruegg, 2014) used on these dairy farms were typical of many larger dairy farms in the upper Midwestern region of the United States. Whereas Staph. aureus was recovered from clinical cases on 20 farms, it was sporadically recovered and accounted for $<4 \%$ of clinical cases that were enrolled in the overall study (Oliveira et al., 2013). Of CNS, Staph. chromogenes and Staph. haemolyticus were most frequently identified. These species have been commonly reported in varying proportions in previous studies that also used genotypic identification, and Staph. chromogenes is typically the most commonly identified species (Taponen et al., 2006; Capurro et al., 2009; Sampimon et al., 2009; Park et al., 2011).

Agreement between identification of species of staphylococci using the $r p o B$ gene and API Staph was approximately $70 \%$, which was similar to previous research (Matthews et al., 1990; Sampimon et al., 2011). Half of the most common Staphylococcus spp. were misidentified using API Staph, indicating the need for a more reliable test when identification at the species level is required. Most importantly, the phenotypic method used in the current study misidentified $20 \%$ of Staph. aureus. The failure to properly identify cows infected with Staph. aureus could delay resolution of a herd problem and dairy professionals should recognize limitations of current testing methodologies. In regions where Staph. aureus is still a significant cause of mastitis, alternative methods of confirming the diagnosis (such as use of chromogenic media or nuc-PCR) should be considered (Virgin et al., 2009; Graber et al., 2013). To identify species of streptococci and SLO, biochemical and phenotypic identification was used because previous researchers have demonstrated full agreement between genotypic and phenotypic identification methods used for identification of Strep. uberis, Strep. dysgalactiae, and Streptococcus agalactiae (Wyder et al., 2011).

Several studies have described antimicrobial susceptibility profiles of bovine mastitis pathogens collected from Wisconsin dairy cows (Makovec and Ruegg, 2003; Pol and Ruegg, 2007a; Oliveira et al., 2012), but no studies have been conducted specifically using isolates collected from larger modern dairy farms that produce the majority of processed milk. The conventional dairy herd population from which isolates were collected in 2004 and 2005 (Pol and Ruegg, 2007a) was composed of smaller Wisconsin dairy herds that were matched with similar organic herds. Whereas neither the previous (Pol and Ruegg, 2007a) nor current studies were specifically designed to estimate prevalence of resistance, comparison of the results is worthwhile. As compared with Pol and Ruegg (2007a), no overall increasing or decreasing trend in the proportion of resistant mastitis pathogens could be discerned. Ceftiofur and cephapirin were the most common IMM products used in the herds enrolled in the current study (Oliveira and Ruegg, 2014), and, similar to Pol and Ruegg, (2007a), almost none of the pathogens examined in the current study were phenotypically resistant to those compounds. Likewise, both studies reported minimal in vitro resistance to oxacillin and the combination of penicillin and novobiocin. Changes in resistance to ampicillin and penicillin were not consistent. As compared with Pol and Ruegg (2007a), the proportion of resistant Staph. aureus was numerically greater in the current study; however, that trend did not apply to CNS (decreased resistance) or Streptococcus spp. (similar proportion of resistant isolates). Some indication exists that resistance of Staph. aureus to penicillin has declined. Makovec and Ruegg (2003) analyzed results of milk samples submitted for antimicrobial resistance testing to the Wisconsin state diagnostic laboratory from 1994 to 2001. Compared with the $20 \%$ of resistant Staph. aureus identified in the present study, they reported that the percentage of Staph. aureus isolates resistant to penicillin decreased from 49 (1994) to $30 \%$ (2001). It is possible that declining resistance to penicillin is related to changes in treatment protocols or is a result of aggressive culling of chronically affected cows in large dairy herds. However, the current study was conducted using isolates collected from Wisconsin dairy herds and it is likely that results may differ from isolates collected in larger herds of other US regions, indicating the need for broader studies throughout the United States.

Pirlimycin was the only antimicrobial that is found in commercially available IMM products used in the United States that demonstrated a considerable proportion of resistant isolates. Pol and Ruegg (2007a) reported that the proportion of pirlimycin resistant organisms was 0 (of 46 Staph. aureus), 18 (of $141 \mathrm{CNS}$ ), and $24 \%$ (of 42 Streptococcus spp.). In the current study, the proportion of Staph. aureus resistant to pirlimycin was greater than in Pol and Ruegg (2007a), but similar proportions of resistance to pirlimycin were detected in the other organisms. Similar to Pol and Ruegg, (2007a), a considerable proportion of CNS and Streptococcus spp. were resistant to sulfadimethoxine and tetracycline. In the United States, sulfadimethoxine is approved for treatment of dairy cattle that are affected with bacterial pneumonia or necrotic pododermatitis. Use of sulfadimethoxine for treatment of any other condition is expressly prohibited by US regulations, but it continues to be used on a small number of farms (Oliveira and Ruegg, 2014). This drug is not expected to achieve a therapeutic concentration in the udder (Baggot, 2006) and it should not be used for mastitis therapy. Whereas 
the systemic administration of tetracycline is allowable under US extra-label guidelines, it has only moderate lipid solubility and the higher $\mathrm{pH}$ of mastitic milk is thought to reduce its antimicrobial activity (Baggot, 2006); thus, use of systemic tetracycline for mastitis therapy should be discouraged.

Many organisms tested using the extended dilution MIC panel were inhibited at concentrations well below the values included in the commercially available panel. Use of custom panel with extended dilutions highlighted that that some organisms have considerable ranges between the $\mathrm{MIC}_{50}$ and $\mathrm{MIC}_{90}$, perhaps indicating a shift in the underlying microbial populations. Given the uncertainty about the validity of non-species- and disease-specific breakpoints, monitoring of the distribution of inhibition may be more useful for detection of selection toward more resistant organisms.

Overall, phenotypic resistance to most selected antimicrobials among Staphylococcus spp. was uncommon and was similar to previous research; this supports previous research indicating that resistance to antimicrobials is not widespread among gram-positive mastitis pathogens (Lüthje and Schwarz, 2006; Rajala-Schultz et al., 2004; Sawant et al., 2009; Sampimon et al., 2011; Oliveira et al., 2012). Ceftiofur was the most common intramammary antimicrobial used by herds enrolled in our study, so it is encouraging that no phenotypic resistance to this antimicrobial was detected in these mastitis pathogens.

Methicillin resistance in Staphylococcus spp. is a cause of both hospital- and community-acquired infections of humans and is a public health concern (Aarestrup and Schwarz, 2006). Phenotypic resistance to oxacillin is one method that is used to detect methicillin resistance of staphylococci. Detection of mecA and $m e c C$ is advised when resistance to methicillin is the subject of research interest, but was not performed on all isolates in the current study because previous studies have established that methicillin resistance is infrequent in Staph. aureus recovered from cases of bovine mastitis (Virgin et al., 2009; Hata et al., 2010; Vanderhaeghen et al., 2010). Only 1 isolate (identified as Staph. epidermidis) demonstrated phenotypic resistance to oxacillin. That isolate was determined to be positive for mecA by PCR testing at the Illinois State Diagnostic Laboratory (Urbana, IL). Resistance to methicillin and multidrug antimicrobial resistance have been previously described in Staph. epidermidis isolated from bovine mastitis (Sawant et al., 2009; Sampimon et al., 2011). Whereas this type of resistance appears to be infrequent, it is theoretically possible that humans and dairy cattle could exchange resistance genes and other researchers have recommended that animals that carry methicillin-resistant staphylococci should be culled (Gentilini et al., 2002).
About $20 \%$ of Staph. aureus and $8 \%$ of CNS demonstrated phenotypic resistance to erythromycin, and most isolates were classified as having intermediate resistance. Erythromycin was not reported to be used in treatments given on these dairy farms (Oliveira and Ruegg, 2014), and the approved IMM erythromycin product has not been available in the United States for many years. However, pirlimycin is used as an IMM product and it is possible that resistance to erythromycin is a result of shared genetic mechanisms.

With the exception of pirlimycin, tetracycline, and sulfadimethoxine, almost all Streptococcus spp. demonstrated susceptibility to most of the selected antimicrobials. According to many researchers, phenotypic resistance to tetracycline is the most common form of resistance among species of streptococci (Erskine et al., 2002; Guérin-Faublée et al., 2002; Makovec and Ruegg, 2003; Bengtsson et al., 2009; Nam et al., 2009). This was confirmed in the present study, where almost half of the isolates demonstrated phenotypic resistance to tetracycline.

Differences among species in resistance to sulfadimethoxine were identified for streptococci. Most Strep. dysgalactiae demonstrated susceptibility to sulfadimethoxine in contrast to widespread resistance of Strep. uberis. Likewise, $68 \%$ of Strep. uberis and $18 \%$ of Strep. dysgalactiae were not inhibited at the greatest concentration of sulfadimethoxine that was tested. Pol and Ruegg (2007a) reported that 21.4\% ( $\mathrm{n}=17)$ of Streptococcus spp. isolated from subclinical mastitis demonstrated resistance to sulfadimethoxine, but the isolates were not identified at the species level. The use of sulfadimethoxine for treatment of mastitis is not permitted by the US Food and Drug Administration, so this use should be actively discouraged.

The proportion of Strep. uberis that have been reported to be phenotypically resistant to enrofloxacin has varied among studies from 3.8 (Rossitto et al., 2002) to $22 \%$ (Roesch et al., 2006). Using the breakpoints for bovine respiratory disease, more than half of Strep. dysgalactiae, Strep. uberis, and SLO were classified as having intermediate resistance to enrofloxacin, and 3 to $7 \%$ were not inhibited at the greatest concentration of enrofloxacin that was tested. Previous researchers have also demonstrated a large proportion of phenotypic resistance of SLO to different antimicrobials (Rossitto et al., 2002; Rajala-Schultz et al., 2004; Pol and Ruegg, 2007a). In spite of US regulations prohibiting the use of enrofloxacin in lactating cows, 1 herd enrolled in the current study used enrofloxacin to treat adult cows experiencing respiratory disease and 8 herds used this drug to treat only calves (which is an allowable usage). However, due to the absence of validated breakpoints for mastitis pathogens, conclusions about trends in the 
development of resistance to enrofloxacin in mastitis pathogens would be speculative.

The criteria used to select the 4 antimicrobial genes included in our study was based on the proportion of isolates that demonstrated phenotypic resistance for the tested antimicrobials, the expected prevalence of these genes among isolates (based on previous research), and the history of antimicrobial usage in the enrolled dairy herds. To date, 10 genes have been identified encoding resistance to macrolides (such as erythromycin), and ermC is the most commonly reported resistance gene (Eady et al., 1993; Jensen et al., 1999; Aarestrup and Schwarz, 2006). At least 38 tetracycline resistance genes have been found, and the tetK gene has been commonly found in species of staphylococci (Schwarz and Chaslus-Dancla, 2001). The tetM gene seems to be identified more commonly in species of staphylococci other than Staph. aureus (Schwarz and Chaslus-Dancla, 2001; Lüthje and Schwarz, 2006).

Of all isolates included in this study, 63\% carried at least 1 of the 4 selected resistance genes. Similar to Sawant et al. (2009), only a few species of staphylococci (mainly Staph. aureus and Staph. chromogenes) carried the erm $C$ gene. As also reported by Sawant et al. (2009), 1 Staph. epidermidis carried ermC; this isolate also carried 2 other genes and was the only isolate that exhibited phenotypic resistance to oxacillin. A study conducted in Germany (Lüthje and Schwarz, 2006) reported that erm $C$ is the most prevalent erm gene recovered from cases of bovine subclinical mastitis caused by CNS and most of the isolates exhibited phenotypic resistance to both erythromycin and pirlimycin. In the present study, whereas phenotypic resistance to pirlimycin was not uncommon, few isolates carried the erm $C$ gene. Isolates that demonstrated phenotypic susceptibility to erythromycin but resistance to pirlimycin are likely to harbor $\operatorname{lnu} B$ gene, which encodes a nucleotidyltransferase that mediates resistance to lincosamides, but not to macrolides (Lüthje and Schwarz, 2006; Schmitt-Van de Leemput and Zadoks, 2007; Apparao et al., 2009b). Apparao (2008) tested the presence of both genes ( $\mathrm{ermC}$ and $\operatorname{lnu} B$ ) in gram-positive pathogens isolated from mastitis before cows received IMM pirlimycin and reported that the prevalence was $8 \%$ for erm $C$ and $3 \%$ for $\operatorname{lnuB}$. The $\ln u B$ gene was not tested in the present study because the $\operatorname{erm} C$ gene was expected to be commonly present in Staphylococcus spp. and also confers resistance to more than 1 antimicrobial. Potentially, phenotypic resistance to pirlimycin and not to erythromycin of Strep. uberis could be related to the presence of $\ln u B$ gene or other unknown mechanisms (Schmitt-Van de Leemput and Zadoks, 2007).

Beta-lactams are important antimicrobials that are often used for prevention and treatment of dairy cattle diseases (Sawant et al., 2009; Pol and Ruegg, 2007b). About 10 to $20 \%$ of Staphylococcus spp. demonstrated phenotypic resistance to penicillin, and the blaZ gene was identified in all resistant Staph. aureus and CNS. However, in agreement with previous research (Haveri et al., 2005), about 40 to $50 \%$ of phenotypically sensitive Staph. aureus and CNS also carried the blaZ gene. There are several possibilities as to why results of phenotypic susceptibility testing may differ from results of genotypic testing. Multiple mechanisms of resistance often exist, but researchers usually only test for a limited number of genes. For example, 21 genes are known to be associated with macrolide-lincosamidestreptogramin resistance. Another possibility could be a mutation of the primer annealing site, as hypothesized by Haveri et al. (2005). However, this is an unlikely explanation because the discrepancy was found in many isolates from different farms and for all genes assessed in the current study. Haveri et al. (2005) suggested that differences in identification of the blaZ gene and the phenotypic development of resistance may be attributable to use of incorrect resistance breakpoints. Those authors suggested that phenotypically susceptible isolates that carry resistance genes should be considered as potentially resistant (Haveri et al., 2005). Breakpoints are discriminatory antimicrobial concentrations used to interpret results of phenotypic susceptibility tests and define isolates as susceptible, intermediate, or resistant (CLSI, 2008). Only ceftiofur, the combination of penicillin and novobiocin, and pirlimycin have validated breakpoints for mastitis, and the application of breakpoints that were defined for other pathogens and different disease conditions for bovine mastitis pathogens has been criticized (Watts and Yancey, 1994; Schwarz et al., 2010). Several studies have demonstrated that in vitro susceptibility tests are not predictive of clinical outcomes (Hoe and Ruegg, 2005; Apparao et al., 2009a; Apparao et al., 2009b) and a clear need exists for more research to better understand the validity and interpretation of both phenotypic and genotypic tests used to determine susceptibility of mastitis pathogens.

Similar to results of the present study, the combination of genotypic and phenotypic resistance to both penicillin and tetracycline has been previously reported for Staph. aureus isolated from bovine IMI (Waage et al., 2002; Haveri et al., 2005). Of all isolates, $43 \%$ carried blaZ and a tet gene; however, this was more prevalent in Staph. aureus compared with other species. Resistance to tetracycline and macrolides is often found in the same mobile unit, Tn1545 (De Leener et al., 2004). Waage et al. (2002) reported that 99 of 107 penicillin and tetracycline resistant Staph. aureus isolates from bovine mastitis occurring in 18 herds belonged to the same strain. Based on these data, Aarestrup 
and Schwarz (2006) hypothesized that the combined penicillin and tetracycline resistance had spread mainly via transmission of a single strain. Clustering of Staph. aureus strains within herds could explain the finds in the present study, but this was not tested, as all of the Staph. aureus that contained the combination of blaZ and tet genes came from 8 herds.

\section{CONCLUSIONS}

The presence of selected resistance genes was not proportional to the occurrence of phenotypic resistance. The majority of the organisms contained at least 1 of the 4 tested resistance genes. Whereas the presence of resistance genes did not correspond with the occurrence of phenotypic resistance, blaZ and tet genes were identified in a large proportion of the isolates. There was a clear discrepancy between results of phenotypic and genotypic testing for antimicrobial resistance, which suggest that a broader selection of genes should be tested for and further research should be directed toward validating resistance breakpoints. Most grampositive mastitis organisms were susceptible to most antimicrobials used for IMM administration but some resistance to drugs used for systemic treatment of mastitis was noted and about $25 \%$ of Staphylococcus spp. were resistant to pirlimycin. All Staph. aureus were susceptible to oxacillin. All isolates were susceptible to both of the cephalosporins that were tested, suggesting that resistance is not emerging to these commonly used IMM compounds. For a few antimicrobials (ampicillin, florfenicol, sulfadimethoxine, and pirlimycin) differences in the proportion of susceptibility, as well $\mathrm{MIC}_{50}$ and $\mathrm{MIC}_{90}$, existed among species of staphylococci and streptococci. This study does not support the evidence of emerging widespread resistance among mastitis pathogens; however, the proportion of some organism resistant to tetracycline and sulfonamides indicate that usage of these drugs should be strongly discouraged.

\section{ACKNOWLEDGMENTS}

Research was supported by USDA Formula Funds and a grant from the American Association of Bovine Practitioners Research Foundation (Auburn, AL). The authors thank Michael O'Connor and Carol Hulland (Wisconsin Veterinary Diagnostic Laboratory, Madison, WI) for technical support.

\section{REFERENCES}

Aarestrup, F. M., and S. Schwarz. 2006. Antimicrobial resistance in staphylococci and streptococci of animal origin. Pages 187-212 in Antimicrobial Resistance in Bacteria of Animal Origin. F. M. Aarestrup, ed. ASM Press, Washington, DC.
Apparao, M. D. 2008. Relationship between lactational antimicrobial therapy and antimicrobial resistance in gram-positive mastitis pathogens recovered from milk samples of primiparous and multiparous dairy cows. MS Thesis. University of Wisconsin, Madison.

Apparao, M. D., L. Oliveira, and P. L. Ruegg. 2009a. Relationship between results of in vitro susceptibility tests and outcomes following treatment with pirlimycin hydrochloride in cows with subclinical mastitis associated with gram-positive pathogens. J. Am. Vet. Med. Assoc. 234:1437-1446.

Apparao, M. D., P. L. Ruegg, A. Lago, S. Godden, R. Bey, and K. Leslie. 2009b. Relationship between in vitro susceptibility test results and treatment outcomes for gram-positive mastitis pathogens following treatment with cephapirin sodium. J. Dairy Sci. 92:2589-2597.

Baggot, J. D. 2006. Principles of antimicrobial drug bioavailability and disposition. Pages 45-79 in Antimicrobial Therapy in Veterinary Medicine. S. Guiguere, J. F. Prescott, J. D. Baggot, R. D. Walker, and P. M. Dowling, ed. Blackwell Publishing, Ames, IA.

Bengtsson, B., H. E. Unnerstad, T. Ekman, K. Artursson, M. NilssonÖst, and K. P. Waller. 2009. Antimicrobial susceptibility of udder pathogens from cases of acute clinical mastitis in dairy cows. Vet. Microbiol. 136:142-149.

Bradley, A. J., K. A. Leach, J. E. Breen, L. E. Green, and M. J. Green. 2007. Survey of the incidence and aetiology of mastitis on dairy farms in England and Wales. Vet. Rec. 160:253-257.

Capurro, A., K. Artursson, K. P. Waller, B. Bengtsson, H. EricssonUnnerstad, and A. Aspán. 2009. Comparison of a commercialized phenotyping system, antimicrobial susceptibility testing, and tuf gene sequence-based genotyping for species-level identification of coagulase-negative staphylococci isolated from cases of bovine mastitis. Vet. Microbiol. 134:327-333.

CLSI (Clinical and Laboratory Standards Institute). 2008. Performance standards for antimicrobial disk and dilution susceptibility tests for bacterial isolated from animals; Approved Standard. 3rd ed. CLSI, Wayne, PA.

CLSI (Clinical and Laboratory Standards Institute). 2013. Performance standards for antimicrobial disk and dilution susceptibility tests for bacteria isolated from animals; Approved Standard. 4th ed. CLSI, Wayne, PA.

Cockerill, F. R. 1999. Genetic methods for assessing antimicrobial resistance. Antimicrob. Agents Chemother. 43:199-212.

De Leener, E., A. Martel, A. Decostere, and F. Haesebrouck. 2004. Distribution of the erm $(B)$ gene, tetracycline resistance genes, and Tn1545-like transposons in macrolide- and lincosamide-resistant enterococci from pigs and humans. Microb. Drug Resist. 10:341-345.

Drancourt, M., and D. Raoult. 2002. rpoB gene sequence-based identification of Staphylococcus species. J. Clin. Microbiol. 40:13331338.

Eady, E. A., J. I. Ross, J. L. Tipper, C. E. Walters, J. H. Cove, and W. C. Noble. 1993. Distribution of genes encoding erythromycin ribosomal methylases and an erythromycin efflux pump in epidemiologically distinct groups of staphylococci. J. Antimicrob. Chemother. 31:211-217.

Erskine, R. J., R. D. Walker, C. A. Bolin, P. C. Bartlett, and D. G. White. 2002. Trends in antibacterial susceptibility of mastitis pathogens during a seven-year period. J. Dairy Sci. 85:1111-1118.

Gentilini, E., G. Denamiel, A. Betancor, M. Rebuelto, M. Rodriguez Fermepin, and R. A. De Torrest. 2002. Antimicrobial susceptibility of coagulase-negative staphylococci isolated from bovine mastitis in Argentina. J. Dairy Sci. 85:1913-1917.

Graber, H. U., S. Pfister, P. Burgener, R. Boss, M. Meylan, and J. Hummerjohann. 2013. Bovine Staphylococcus aureus: Diagnostic properties of specific media. Res. Vet. Sci. 95:38-44.

Guérin-Faublée, V., F. Tardy, C. Bouveron, and G. Carret. 2002. Antimicrobial susceptibility of Streptococcus species isolated from clinical mastitis in dairy cows. Int. J. Antimicrob. Agents 19:219-226.

Hata, E., K. Katsuda, H. Kobayashi, I. Uchida, K. Tanaka, and M. Eguchi. 2010. Genetic variation among Staphylococcus aureus strains from bovine milk and their relevance to methicillin-resistant isolates from humans. J. Clin. Microbiol. 48:2130-2139. 
Haveri, M., S. Suominen, L. Rantala, T. Honkanen-Buzalski, and S. Pyörälä. 2005. Comparison of phenotypic and genotypic detection of penicillin G resistance of Staphylococcus aureus isolated from bovine intramammary infection. Vet. Microbiol. 106:97-102.

Hoe, F. G. H., and P. L. Ruegg. 2005. Relationship between antimicrobial susceptibility of clinical mastitis pathogens and treatment outcome in cows. J. Am. Vet. Med. Assoc. 227:1461-1468.

Jensen, L. B., N. Frimodt-Moller, and F. M. Aarestrup. 1999. Presence of erm gene classes in gram-positive bacteria of animal and human origin in Denmark. FEMS Microbiol. Lett. 170:151-158.

Lüthje, P., and S. Schwarz. 2006. Antimicrobial resistance of coagulase-negative staphylococci from bovine subclinical mastitis with particular reference to macrolide-lincosamide resistance phenotypes and genotypes. J. Antimicrob. Chemother. 57:966-969.

Makovec, J. A., and P. L. Ruegg. 2003. Antimicrobial resistance of bacteria isolated from dairy cow milk samples submitted for bacterial culture: 8,905 samples (1994-2001). J. Am. Vet. Med. Assoc. $222: 1582-1589$.

Matthews, K. R., S. P. Oliver, and S. H. King. 1990. Comparison of Vitek Gram-positive identification system with API Staph-Trac system for species identification of staphylococci of bovine origin. J. Clin. Microbiol. 28:1649-1651.

McDougall, S., D. G. Arthur, M. A. Bryan, J. J. Vermunt, and A. M. Weir. 2007. Clinical and bacteriological response to treatment of clinical mastitis with one of three intramammary antibiotics. N. Z. Vet. J. 55:161-170.

Nam, H.-M., S.-K. Lim, H.-M. Kang, J.-M. Kim, J.-S. Moon, K.-C Jang, Y.-S. Joo, M.-I. Kang, and S.-C. Jung. 2009. Antimicrobial resistance of streptococci isolated from mastitic bovine milk samples in Korea. J. Vet. Diagn. Invest. 21:698-701.

National Mastitis Council. 1999. Laboratory Handbook on Bovine Mastitis. National Mastitis Council, Verona, WI.

Neu, H. C. 1992. An update on fluoroquinolones. Curr. Opin. Infect. Dis. 5:755-763.

Oliveira, L., C. Hulland, and P. L. Ruegg. 2013. Characterization of clinical mastitis occurring in cows on 50 large dairy herds in Wisconsin. J. Dairy Sci. 96:7538-7549.

Oliveira, L., H. Langoni, C. Hulland, and P. L. Ruegg. 2012. Minimum inhibitory concentrations of Staphylococcus aureus recovered from clinical and subclinical cases of bovine mastitis. J. Dairy Sci. 95:1913-1920.

Oliveira, L., and P. L. Ruegg. 2014. Treatments of clinical mastitis occurring in cows on 51 large dairy herds in Wisconsin. J. Dairy Sci. $97: 5426-5436$.

Park, J. Y., L. K. Fox, K. S. Seo, M. A. McGuire, Y. H. Park, F. R. Rurangirwa, W. M. Sischo, and G. A. Bohach. 2011. Comparison of phenotypic and genotypic methods for the species identification of coagulase-negative staphylococcal isolates from bovine intramammary infections. Vet. Microbiol. 147:142-148.

Pinzón-Sanchez, C., and P. L. Ruegg. 2011. Risk factors associated with short-term post-treatment outcomes of clinical mastitis. J. Dairy Sci. 94:3397-3410.

Pol, M., and P. L. Ruegg. 2007a. Relationship between antimicrobial drug usage and antimicrobial susceptibility of gram-positive mastitis pathogens. J. Dairy Sci. 90:262-273.

Pol, M., and P. L. Ruegg. 2007b. Treatment practices and quantification of antimicrobial drug usage in conventional and organic dairy farms in Wisconsin. J. Dairy Sci. 90:249-261.

Rajala-Schultz, P. J., K. L. Smith, J. S. Hogan, and B. C. Love. 2004 Antimicrobial susceptibility of mastitis pathogens from first lactation and older cows. Vet. Microbiol. 102:33-42.

Roesch, M., V. Perreten, M. G. Doherr, W. Schaeren, M. Schällibaum, and J. W. Blum. 2006. Comparison of antibiotic resistance of udder pathogens in dairy cows kept on organic and on conventional farms. J. Dairy Sci. 89:989-997.

Rossitto, P. V., L. Ruiz, Y. Kikuchi, K. Glenn, K. Luiz, J. L. Watts, and J. S. Cullor. 2002. Antibiotic susceptibility patterns for environmental streptococci isolated from bovine mastitis in central California dairies. J. Dairy Sci. 85:132-138.

SAS Institute. 2011. SAS/STAT User's Guide. Version 9.3. SAS Institute Inc., Cary, NC.
Saini, V., J. T. McClure, D. Léger, S. Dufour, A. G. Sheldon, D. T. Scholl, and H. W. Barkema. 2012. Antimicrobial use on Canadian dairy farms. J. Dairy Sci. 95:1209-1221.

Sambrook, J., and D. W. Russell. 2001. Molecular Cloning: A Laboratory Manual. 3rd ed. Cold Spring Harbor Laboratory Press, Cold Spring Harbor, NY.

Sampimon, O. C., T. J. G. M. Lam, D. J. Mevius, Y. H. Schukken, and R. N. Zadoks. 2011. Antimicrobial susceptibility of coagulasenegative staphylococci isolated from bovine milk samples. Vet. Microbiol. 150:173-179.

Sampimon, O. C. R. N. Zadoks, S. De Vliegher, K. Supré, F. Haesebrouck, H. W. Barkema, J. Sol, and T. J. G. M. Lam. 2009. Performance of API Staph ID 32 and Staph-Zym for identification of coagulase-negative staphylococci isolated from bovine milk samples. Vet. Microbiol. 136:300-305.

Sawant, A. A., B. E. Gillespie, and S. P. Oliver. 2009. Antimicrobial susceptibility of coagulase-negative Staphylococcus species isolated from bovine milk. Vet. Microbiol. 134:73-81.

Schmitt-Van de Leemput, E., and R. N. Zadoks. 2007. Genotypic and phenotypic detection of macrolide and lincosamide resistance in Streptococcus uberis. J. Dairy Sci. 90:5089-5096.

Schwarz, S., and E. Chaslus-Dancla. 2001. Use of antimicrobials in veterinary medicine and mechanisms of resistance. Vet. Res. $32: 201-225$.

Schwarz, S., P. Silley, S. Simjee, N. Woodford, E. van Duijkeren, A P. Johnson, and W. Gaastra. 2010. Editorial: Assessing the antimicrobial susceptibility of bacteria obtained from animals. J. Antimicrob. Chemother. 65:601-604.

Sutcliffe, J., T. Grebe, A. Tait-Kamradt, and L. Wondrack. 1996. Detection of erythromycin-resistant determinants by PCR. Antimicrob. Agents Chemother. 40:2562-2566.

Taponen, S., H. Simojoki, M. Haveri, H. D. Larsen, and S. Pyorala. 2006. Clinical characteristics and persistence of bovine mastitis caused by different species of coagulase-negative staphylococci identified with API or AFLP. Vet. Microbiol. 115:199-207.

USDA. 2007. Part V: Changes in Dairy Cattle Health and Management Practices in the United States, 1996-2007. National Animal Health Monitoring Service, ed. USDA, Washington, DC.

Vanderhaeghen, W., T. Cerpentier, C. Adriaensen, J. Vicca, K. Hermans, and P. Butaye. 2010. Methicillin-resistant Staphylococcus aureus (MRSA) ST398 associated with clinical and subclinical mastitis in Belgian cows. Vet. Microbiol. 144:166-171.

Virgin, J. E., T. M. Van Slyke, J. E. Lombard, and R. N. Zadoks. 2009. Short communication: Methicillin-resistant Staphylococcus aureus detection in US bulk tank milk. J. Dairy Sci. 92:4988-4991.

Waage, S., J. Bjorland, D. A. Caugant, H. Oppegaard, T. Tollersrud, T. Mork, and F. M. Aarestrup. 2002. Spread of Staphylococcus aureus resistant to penicillin and tetracycline within and between dairy herds. Epidemiol. Infect. 129:193-202.

Walker, R. D. 2006. Antimicrobial susceptilbity testing methods and interpretation of results. Pages 11-26 in Antimicrobial Therapy in Veterinary Medicine. S. Guiguere, J. F. Prescott, J. D. Baggot, R. D. Walker, and P. M. Dowling, ed. Blackwell Publishing, Ames, IA.

Walther, C., and V. Perreten. 2007. Letter to the editor: Methicillinresistant Staphylococcus epidermidis in organic milk production. J. Dairy Sci. 90:5351.

Watts, J. L., and R. J. Yancey Jr.. 1994. Identification of veterinary pathogens by use of commercial identification systems and new trends in antimicrobial susceptibility testing of veterinary pathogens. Clin. Microbiol. Rev. 7:346-356.

Woodford, N., and A. Sundsfjord. 2005. Molecular detection of antibiotic resistance: When and where? J. Antimicrob. Chemother. $56: 259-261$.

Wyder, A. B., R. Boss, J. Naskova, T. Kaufmann, A. Steiner, and H. U. Graber. 2011. Streptococcus spp. and related bacteria: Their identification and their pathogenic potential for chronic mastitisA molecular approach. Res. Vet. Sci. 91:349-357. 